\title{
Stochastically Globally Exponential Stability of Stochastic Impulsive Differential Systems with Discrete and Infinite Distributed Delays Based on Vector Lyapunov Function
}

\author{
Xiaoyan Liu ${ }^{1,2}$ and Quanxin Zhu $\mathbb{D}^{1}$ \\ ${ }^{1}$ MOE-LCSM, School of Mathematics and Statistics, Hunan Normal University, Changsha, Hunan 410081, China \\ ${ }^{2}$ School of Information Science and Engineering, Chengdu University, Chengdu 610106, China \\ Correspondence should be addressed to Quanxin Zhu; zqx22@126.com
}

Received 23 July 2020; Revised 19 September 2020; Accepted 25 September 2020; Published 1 December 2020

Academic Editor: Shuping He

Copyright (C) 2020 Xiaoyan Liu and Quanxin Zhu. This is an open access article distributed under the Creative Commons Attribution License, which permits unrestricted use, distribution, and reproduction in any medium, provided the original work is properly cited.

\begin{abstract}
This paper deals with stochastically globally exponential stability (SGES) for stochastic impulsive differential systems (SIDSs) with discrete delays (DDs) and infinite distributed delays (IDDs). By using vector Lyapunov function (VLF) and average dwell-time (ADT) condition, we investigate the unstable impulsive dynamics and stable impulsive dynamics of the suggested system, and some novel stability criteria are obtained for SIDSs with DDs and IDDs. Moreover, our results allow the discrete delay term to be coupled with the nondelay term, and the infinite distributed delay term to be coupled with the nondelay term. Finally, two examples are given to verify the effectiveness of our theories.
\end{abstract}

\section{Introduction}

During the past few years, stochastic differential systems (SDSs) have been paid a great deal of attention in various fields. For example, there have been a number of works on the stability of SDSs (e.g., see [1-6]), and such a topic is of great significance in many practical applications. On the other hand, delay problems are often encountered in the chemical industry system, neural network system, and other systems. As a result, the stability analysis problem of SDSs with delays was studied in [2-12], where the delays were divided into constant delays, time-varying delays, and distributed delays. For a kind of SDSs with DDs and IDDs, there are many researchers to pay much attention on this topic, SDSs with DDs and IDDs were applied to study neural network systems [13-15], especially. However, these modes do not include some dynamic phenomena with impulses even if they are indeed important in practice.

Systems with impulses have been widely applied in practice. For instance, they are often used to describe dynamic processes that mutate at successive times
$[7,8,11,12,16-20]$. In the past few decades, addressed system was studied extensively (e.g., see $[16,19])$ and it was found that impulsive systems can contribute the exponential stability of SDSs (e.g., see $[8,11,12])$. The impulse one can not only cause complex dynamic behaviors such as instability, but also stabilize the unstable dynamic system. How to use the appropriate impulse control to stabilize the unstable SDSs or let impulses play a negative role on the stable system is of great significance. This paper aims to study these interesting topics for SIDSs with DDs and IDDs.

In order to deal with the stability of systems with DDs and IDDs, there have appeared many methods such as the fixed point theory, Lyapunov-Krasovskii function or the scalar Lyapunov function. For example, the Lyapunov -Krasovskii function and matrix inequality method were used in [13]; Chen et al. in [15] employed the fixed point theorem; Huang and Cao in [14] applied the Lyapunov functional method and the semimartingale convergence theorem. However, until now, there have been essentially no results to deal with SIDSs with DDs and IDDs by using the vector Lyapunov method. To investigate the stability issue, 
two new methods were recently proposed: the VLF method $[9,12,19-23]$ and the ADT method $[18,24]$. The ADT effectively limits the impulse and could promote the stability of the system. By using VLF, inequality techniques, and impulse conditions, some useful exponential stability criteria are obtained. As a feasible alternative to scalar Lyapunov function, VLF has attracted more and more attention in recent years (e.g., see [9]). In [25], VLF was first introduced and widely used in various fields owing to its outstanding advantages. In terms of construction, the theory of VLF provides a more flexible method for dealing with the complexity of SIDSs (e.g., see $[19,26])$. The real reason is that the theory of VLF can reduce the dimension and reduce the requirement of system component (e.g., see [26]). Therefore, there are many related results reported on the vector Lyapunov function method (e.g., see [9, 12, 19-23]). However, the joint system with IDDs of stochastic impulsive and DDs has not been solved, which greatly limits the effectiveness of VLF.

Motivated by the above discussions, we study SGES of SIDS with DDs and IDDs by using ADT condition and VLF. We consider two cases: unstable impulse dynamics and stable impulsive dynamics. For these two cases, some sufficient conditions are established for SIDS with DDs and IDDs based on the strength of VLF and ADT condition. Moreover, the results show that continuous SDSs with DDs and IDDs are stable and the impulsive one is unstable, according to the relationship between ADT and impulse, a lower bound of ADT is given to the mixed system is exponential stability. When continuous SIDS with DDs and IDDs are not stable, the impulsive effect can stabilize the system successfully under the upper bound condition of the given ADT.

There are three contributions to the paper. (1) To the best of our knowledge, there have been no studies on the stability of SIDS with DDs and IDDs by VLF. (2) The discrete delay term is coupled with the nondelay term and the infinitely distributed delay term is coupled with the nondelay term. It should be mentioned that the comparison principle was used
$[19,20,23]$ and the components of VLF were separate, but the coupling of distributed delay term with nondelay term was not considered in $[9,12]$. (3) The third is infinitely distributed delay: due to its infinite nature, we deal with it by the construction formula $\sum_{j=1}^{n} \int_{0}^{\infty} k_{i j}(s) \exp \left(\eta_{i} s\right) \alpha_{j} \mathrm{~d} s$ and $\sum_{j=1}^{n} \int_{0}^{\infty} k_{i j}(s) \exp \left(-\eta_{i} s\right) \alpha_{j} \mathrm{~d} s$. Thus, our results are innovative than those in $[9,19,20,23]$.

The remainder of this paper is organized as follows. In the second part, the model and the preliminary knowledge are introduced. Two novel stability criteria are established for the stochastic impulsive systems with DDs and IDDs in the third part. In the fourth part, two examples are given to verify the correctness of our results.

\section{Preliminaries}

Through the paper, no special instructions, we will use the following instructions.

$w(t)$ is an $\mathbb{R}^{m}$-valued Brownian motion defined on a complete probability space $\left(\Omega, \mathscr{F},\left\{\mathscr{F}_{t}\right\}_{t \geq t_{0}}, P\right) . N_{>0}$ represents the set of positive integer and $\mathbb{R}$ denotes the real number. For a give $t_{0} \geq 0$, let $\mathbb{R}_{\geq t_{0}}:=\left(t_{0},+\infty\right]$. Given $a$, $b \in \mathbb{R}^{n}, a>b$ if $a_{i}>b_{i}$, for all $i \in \iota=\{1, \ldots, n\}$. Define $(a, b):=\left(a^{T}, b^{T}\right)^{T}$. Given a vector or matrix $C$, its transpose is denoted by $C^{\mathrm{T}}$. $A \backslash D:=\{z: z \in A, z \in D\}$ for two given sets $A$ with $D . \operatorname{tr}[B]$ represents the trace of the matrix $B$, where $B=B^{T} \in \mathbb{R}^{n \times n}$. Let $|$.$| be the Euclidean norm and E$ present the vector that all the components are 1 . I means identity matrix. For a given function $p: \mathbb{R}_{\geq t_{0}} \longrightarrow \mathbb{R}^{n}$ and the initial time $-\infty<t \leq t_{0}$, define $\|p\|_{\infty}:=\sup _{t \leq t_{0}}|p(t)|$. Given a function $g: \mathbb{R} \longrightarrow \mathbb{R}$, denote $g\left(t^{-}\right):=\limsup _{s \rightarrow 0^{-}} g(t+s)$. A function $\alpha: \mathbb{R}_{\geq 0} \longrightarrow \mathbb{R}_{\geq 0}$ is of class $\mathscr{C} \mathscr{K}_{\infty}$ if it is of class $\mathscr{K}_{\infty}$ and concave. A function $\gamma: \mathbb{R}_{\geq 0} \times \mathbb{R}_{\geq 0} \longrightarrow \mathbb{R}_{\geq 0}$ is of class $\mathscr{K} \mathscr{L}$, if $\gamma(s, t)$ is of class $\mathscr{K}$ for each fixed $t \geq 0$ and decreases to zero as $t \longrightarrow \infty$ for each fixed $s \geq 0$. The inverse of the function $\beta$ is denoted by $\beta^{-1}$. IDDs:

$$
\left\{\begin{array}{l}
\mathrm{d} x(t)=f\left(t, x_{t}, \int_{0}^{\infty} k(s) x(t-s) \mathrm{d} s\right) \mathrm{d} t+g\left(t, x_{t}, \int_{0}^{\infty} k(s) x(t-s) \mathrm{d} s\right) \mathrm{d} w(t), \quad t \in \mathbb{R}_{\geq} t_{0} \backslash \mathscr{T} \\
\left.\Delta x(t)\right|_{t=t_{k}}=x\left(t_{k}\right)-x\left(t_{k}^{-}\right)=h_{k}\left(t_{k}, x\left(t_{k}^{-}\right)\right), \quad t \in \mathscr{T}, k \in N_{>0}, \\
x(t)=\xi(t), \quad t \in\left(-\infty, t_{0}\right]
\end{array}\right.
$$

where $x(t) \in \mathbb{R}^{n}$ is the system state, $x_{t}:=x(t-\tau), \tau$ is a bounded and positive constant. $\mathscr{T}:=\left\{t_{0}, t_{1}, \ldots, t_{n}\right\}$ is a impulsive time sequence satisfying $0 \leq t_{0} \leq t_{1} \leq \cdots \leq t_{n}$. The initial function $\xi:\left[-\infty, t_{0}\right] \longrightarrow \mathbb{R}^{n}$ is a $\mathscr{F}_{t_{0}^{-}}$adapted continuous stochastic variable with finite $\mathbb{E}\left[\|\xi\|_{\tau}^{p}\right]$. For all $k \in N_{>0}$, the function $f: \mathbb{R}_{\geq t_{0}} \times \mathbb{R}^{n} \times \mathbb{R}^{n} \longrightarrow \mathbb{R}^{n}$, $g: \mathbb{R}_{\geq t_{0}} \times \mathbb{R}^{n} \times \mathbb{R}^{n} \longrightarrow \mathbb{R}^{n \times m}$, and $h_{k}: \mathbb{R}_{\geq t_{0}} \times \mathbb{R}^{n} \longrightarrow \mathbb{R}^{n}$ are assumed to be Lipschitz and Borel measurable. For the aim of stability, we assume that $f(t, 0,0) \equiv 0$ and $g(t, 0,0) \equiv 0$, and thus $x(t) \equiv 0$ is a trivial solution of system (1). As a usual, we assume with no emphasis on conditions that there exists a unique global solution $x(t, \xi)$ for the initial value $\xi$.

In this paper, we always assume that $K:=\left[\int_{0}^{\infty} k_{i j} \mathrm{~d} s\right]_{n \times n}$ is meaningful. That is, the delay kernels $k_{i j}:[0,+\infty) \rightarrow[0,+\infty)$ are real-valued nonnegative continuous functions and satisfy $\int_{0}^{\infty} e^{\mu s} k_{i j}(s) \mathrm{d} s<\infty$, where $\mu$ is a positive number.

Definition 1 (see [6]): For arbitrary $\varepsilon \in(0,1)$, if there is $\varrho \in \mathscr{K} \mathscr{L}, x\left(t_{0}\right) \in \mathbb{R}^{n}, t \in \mathbb{R}_{\geq t_{0}}, M \geq 0, b>0$ such that 


$$
P\left\{|x(t)| \leq \varrho\left(\left|x\left(t_{0}\right)\right|, t-t_{0}\right)\right\} \geq 1-\varepsilon .
$$

Then, system (1) is stochastically globally asymptotically stable. Furthermore, if $\varrho(a, t):=M a^{2} e^{-b t}$, then system (1) is stochastically globally exponentially stable (SGES).

Definition 2 (see [11, 24]): For an impulsive sequence $\left\{t_{k}\right\}_{k \in N}, N(t, s)$ shows the number of impulses that occur in the half-open interval $(s, t]$. If

$$
\frac{t-s}{\tau_{c}}-N_{0} \leq N(t, s) \leq \frac{t-s}{\tau_{c}}+N_{0}
$$

for $N_{0}>0, \tau_{c}>0$, then $\tau_{c}$ and $N_{0}$ are called the average dwell-time (ADT) and the elasticity number, respectively.

Definition 3 (see [9]): Let $\mathscr{C}^{1,2}$ denote the family of the nonnegative functions $V_{i}(t, x): \mathbb{R}_{\geq 0} \times \mathbb{R}^{n} \longrightarrow \mathbb{R}_{\geq 0}$ that are continuously twice differentiable in $x$ and once in $t, n$ is bounded. Then, for any $\mathscr{C}^{1,2}$, the operator of $\mathscr{L} V_{i}\left(t, x_{t}, \int_{0}^{\infty} k(s) x(t-s) \mathrm{d} s\right): \mathbb{R}_{\geq 0} \times \mathbb{R}^{n} \times \mathbb{R}^{n} \longrightarrow \mathbb{R}_{\geq 0}$, $i \in \iota$ is defined as

$$
\begin{aligned}
\mathscr{L} V_{i}\left(t, x_{t}, \int_{0}^{\infty} k(s) x(t-s) \mathrm{d} s\right)= & \frac{\partial V_{i}(t, x(t))}{\partial t}+\frac{\partial V_{i}(t, x(t))}{\partial x} f\left(t, x_{t}, \int_{0}^{\infty} k(s) x(t-s) \mathrm{d} s\right) \\
& +\frac{1}{2} \operatorname{tr}\left[g^{T}\left(t, x_{t}, \int_{0}^{\infty} k(s) x(t-s) \mathrm{d} s\right) \frac{\partial V_{i}(t, x(t))}{\partial x^{2}} g\left(t, x_{t}, \int_{0}^{\infty} k(s) x(t-s) \mathrm{d} s\right)\right] .
\end{aligned}
$$

Define $V(t, x(t)):=\left(V_{1}(t, x(t)), \ldots, V_{n}(t, x(t))\right)$. In particular, if $n=1$, then $V$ becomes a scalar.

Let $\Psi: \mathbb{R}_{\geq t_{0}} \longrightarrow \mathbb{R}_{\geq t_{0}}$ be a continuous function. Then, the upper Dini derivative of $\Psi(t)$ is defined as $D^{+} \Psi(t):=\limsup _{s \rightarrow 0^{+}} \Psi(t+s)-\Psi(t) / s$. In particular, if $\mathbb{E}\left[\mathscr{L} V_{i}\left(t, x(t), x_{t}\right)\right]$ is continuous, then it follows from [27] that $\mathbb{E}\left[\mathscr{L} V_{i}\left(t, x(t), x_{t}\right)\right]=D^{+}\left[\mathbb{E} V_{i}\left(t, x(t), x_{t}\right)\right]$.

Definition 4 (see [28]) Let off-diagonal elements of the matrix $A=\left(a_{i j}\right)_{n \times n}$ be nonpositive. If each of the following statements holds, then $A$ is a nonsingular M-matrix.

(1) If the diagonal elements of A are all positive, then there exists a positive vector $a$ such that $A a>0$ or $A^{\mathrm{T}} a>0$.

(2) $A=C-M, \quad \rho\left(C^{-1} M\right)<1$, where $\quad M \geq 0$, $C=\operatorname{diag}\left\{c_{1}, c_{2}, \ldots, c_{n}\right\}$ and $\rho(\cdot)$ is the spectral radius of the matrix $(\cdot)$.
For a nonsingular M-matrix $A$, it can denote $\Omega_{M}(A) \triangleq\left\{z \in R^{n} \mid A z>0, z>0\right\}$.

\section{Main Results}

In this section, we will establish the SGES of system (1) with destabilizing impulses.

Theorem 1. For system (1), assume that there is locally Lipschitz Lyapunov function $V_{i}: \mathbb{R}_{\geq 0} \times \mathbb{R}^{n} \longrightarrow \mathbb{R}_{\geq 0}$, $i \in l$, $r>1, \quad \zeta E \geq E, \quad v \in \mathscr{K}_{\infty}, \quad \varsigma \in \mathscr{C} \mathscr{K}_{\infty}$, positive matrices $\mu:=\operatorname{diag}\left(\mu_{1}, \mu_{2}, \ldots, \mu_{n}\right), \zeta:=\operatorname{diag}\left(\zeta_{1}, \zeta_{2}, \ldots, \zeta_{n}\right)$ and nonnegative matrices $P:=\left[p_{i j}\right]_{n \times n}, Q:=\left[q_{i j}\right]_{n \times n}, O:=\left[o_{i j}\right]_{n \times n}$, where $K:=\left[\int_{0}^{\infty} k_{i j}(s) d s\right]_{n \times n}$ is continuous function and $k_{i j}(s) \geq 0$ such that the following conditions:

(i) $i \in l, t \in \mathbb{R}_{\geq t_{0}}, v(|x(t)|) \leq V_{i}(t) \leq \varsigma(|x(t)|)$.

(ii) $i \in \iota, t \in \mathbb{R}_{\geq t_{0}} \backslash \mathscr{T}$,

$$
\begin{aligned}
\mathscr{L} V_{i}\left(t, x(t), x_{t}, \int_{0}^{\infty} k(s) x(t-s) \mathrm{d} s\right) \leq & -\mu_{i} V_{i}(t)+\sum_{j=1}^{n} p_{i j}\left(V_{i}(t)\right)^{1 / r^{\prime}}\left(V_{j}(t)\right)^{1 / r}+\sup _{-\tau \leq \theta \leq 0} \sum_{j=1}^{n} q_{i j}\left(V_{i}(t)\right)^{1 / r^{\prime}}\left(V_{j}(t+\theta)\right)^{1 / r} \\
& +\sum_{j=1}^{n} \int_{0}^{\infty} k_{i j}(s)\left(V_{i}(t)\right)^{1 / r^{\prime}}\left(V_{j}(t-s)\right)^{1 / r} \mathrm{~d} s
\end{aligned}
$$

where $\mu-P-Q-K$ is a nonsingular $M$-matrix, $\theta \in[-\tau, 0], r^{\prime}=\left(1-r^{-1}\right)^{-1}$.

(iii) $i \in \iota$ and $k \in N_{>0}$,

$$
V_{i}\left(t_{k}\right) \leq \zeta_{i} V_{i}\left(t_{k}^{-}\right)+\left(\sum_{j=1}^{n} o_{i j}\right)\left(V_{i}\left(t_{k}^{-}\right)\right)^{1 / r^{\prime}}\left(V_{j}\left(t_{k}^{-}\right)\right)^{1 / r}
$$


(iv) $\tau_{c}>\max _{i \in t} \ln \left(\zeta_{i}+\sum_{j=1}^{n} o_{i j}\right) / \lambda$, where $\lambda \in(0, \bar{\lambda})$, $V_{i}(t) \stackrel{i \in l}{=} V_{i}(t, x(t))$,

$$
\bar{\lambda}:=\max \left\{\eta_{i}>0: r \alpha_{i} \eta_{i}-\mu_{i} \alpha_{i}+\sum_{j=1}^{n} p_{i j} \alpha_{j}+\sum_{j=1}^{n} q_{i j} \alpha_{j} \exp \left(\tau \eta_{i}\right)+\sum_{j=1}^{n} \int_{0}^{\infty} k_{i j}(s) \exp \left(\eta_{i} s\right) \alpha_{j} \mathrm{~d} s<0\right\},
$$

where $i \in \iota, \alpha \in \Omega_{M}(\mu-P-Q-K)$, with $\min _{1 \leq i \leq n}\left\{\alpha_{i}\right\} \geq 1$, $\bar{\alpha}=\max \left\{\alpha_{i}\right\}$.

Then, system (1) is SGES.
Proof. By taking expectation on both sides of (5) and (6) and using Holder inequality in [6], we have

$$
\begin{aligned}
& \mathbb{E} \mathscr{L} V_{i}\left(t, x(t), x_{t}, \int_{0}^{\infty} k(s) x(t-s) \mathrm{d} s\right) \leq-\mu_{i} \mathbb{E} V_{i}(t)+\sum_{j=1}^{n} p_{i j}\left(\mathbb{E} V_{i}(t)\right)^{1 / r^{\prime}}\left(\mathbb{E} V_{j}(t)\right)^{1 / r}+\sup _{-\tau \leq \theta \leq 0} \sum_{j=1}^{n} q_{i j}\left(\mathbb{E} V_{i}(t)\right)^{1 / r^{\prime}}\left(\mathbb{E} V_{j}(t+\theta)\right)^{1 / r} \\
&+\sum_{j=1}^{n} \int_{0}^{\infty} k_{i j}(s)\left(\mathbb{E} V_{i}(t)\right)^{1 / r^{\prime}}\left(\mathbb{E} V_{j}(t-s)\right)^{1 / r} \mathrm{~d} s, \quad i \in \iota, t \in \mathbb{R}_{\geq t_{0}} \backslash \mathscr{T} . \\
& \mathbb{E} V_{i}\left(t_{k}\right) \leq \zeta_{i} \mathbb{E} V_{i}\left(t_{k}^{-}\right)+\left(\sum_{j=1}^{n} o_{i j}\right)\left(\mathbb{E} V_{i}\left(t_{k}^{-}\right)\right)^{1 / r^{\prime}}\left(\mathbb{E} V_{j}\left(t_{k}^{-}\right)\right)^{1 / r}, \quad i \in \iota \text { and } k \in N_{>0} .
\end{aligned}
$$

Next, we divide the proof into three steps.

$$
\mu_{i} \alpha_{i}-\sum_{j=1}^{n} p_{i j} \alpha_{j}-\sum_{j=1}^{n} q_{i j} \alpha_{j}-\sum_{j=1}^{n} \int_{0}^{\infty} k_{i j}(s) \alpha_{j} \mathrm{~d} s>0, \quad i \in \iota
$$

If $\mu-P-Q-K$ is a nonsingular M-matrix, there is $\alpha \in \Omega_{M}(\mu-P-Q-K)$ with $\min _{1 \leq i \leq n}\left\{\alpha_{i}\right\} \geq 1$ such that

For any given $i$, let

$$
H_{i}(\eta)=-r \alpha_{i} \eta+\mu_{i} \alpha_{i}-\sum_{j=1}^{n} p_{i j} \alpha_{j}-\sum_{j=1}^{n} q_{i j} \alpha_{j} \exp (\tau \eta)-\sum_{j=1}^{n} \int_{0}^{\infty} k_{i j}(s) \exp (\eta s) \alpha_{j} \mathrm{~d} s .
$$

Obviously, it follows from (11) that $H_{i}(0)>0$ and $H_{i}(\infty)=-\infty$, where $\eta \longrightarrow \infty$. On the other hand, we have

$$
H_{i}^{\prime}(\eta)=-r \alpha_{i}-\tau \sum_{j=1}^{n} q_{i j} \alpha_{j} \exp (\tau \eta)-\sum_{j=1}^{n} \int_{0}^{\infty} s k_{i j}(s) \exp (\eta s) \alpha_{j} \mathrm{~d} s<0
$$

Thus, there is a unique constant $\eta_{i}>0$ such that $H_{i}\left(\eta_{i}\right)=0$. Letting $\bar{\lambda}=\max _{1 \leq i \leq n} \eta_{i}>0$, there is a constant $\lambda$ such that $\lambda \in(0, \bar{\lambda})$.

Step 2. Next, we need to prove that for $i \in \iota$ and $\lambda \in(0, \bar{\lambda})$,

$$
\mathbb{E}\left[V_{i}(t)\right] \leq \mathbb{E}\left[V\left(t_{0}\right)\right] \bar{\mu}^{r N\left(t, t_{0}\right)} \bar{\alpha}^{r} \exp \left(-r \lambda\left(t-t_{0}\right)\right),
$$

where $V\left(t_{0}\right)=\sum_{i=1}^{n} V_{i}\left(t_{0}\right), \quad \bar{\mu}=\max _{i \in \Lambda}\left\{\zeta_{i}+\sum_{j=1}^{n} o_{i j}\right\}$ and $\bar{\alpha}:=\max _{i \in l} \alpha_{i}$. Clearly, (13) holds for $t=t_{0}$. For simplicity, let $W_{i}\left(t-t_{0}\right)=\left[\mathbb{E} V\left(t_{0}\right)\right]^{1 / r} \bar{\mu}^{N\left(t, t_{0}\right)} \alpha_{i}$ $\exp \left(-\lambda\left(t-t_{0}\right)\right), U_{i}(t)=\left[\mathbb{E} V_{i}(t)\right]^{1 / r}$.

It is easy to check that (13) is equivalent to the following: 


$$
\left[\mathbb{E} V_{i}(t)\right]^{1 / r} \leq \max _{i \in l}\left\{\left[\mathbb{E} V\left(t_{0}\right)\right]^{1 / r} \bar{\mu}^{N\left(t, t_{0}\right)} \alpha_{i} \exp \left(-\lambda\left(t-t_{0}\right)\right)\right\}=: \max _{i \in l}\left\{W_{i}\left(t-t_{0}\right)\right\} .
$$

Now suppose that (13) is not valid in some interval, then there are two cases:

Case 1. (13) is not true at the nonimpulse point of the certain interval;

Case 2. (13) does not hold at the impulse point of the certain interval.

For Case 1 , there exists a $k$ such that $U_{i}(t) \leq W_{i}\left(t-t_{0}\right)$ holds for all $t \in\left[t_{0}, t_{k}\right]$ and $i \in \iota$, and $U_{i}(t) \leq W_{i}\left(t-t_{0}\right)$ is not true for $i \in \iota$ and $t \in\left(t_{k}, t_{k+1}\right)$. Define

$$
t^{*}:=\inf \left\{t \in\left(t_{k}, t_{k+1}\right): U_{i}(t)>W_{i}\left(t-t_{0}\right)\right\} .
$$

Noting that $U_{i}(t)$ and $W_{i}\left(t-t_{0}\right)$ are continuous for $t \in \mathbb{R}_{\geq t_{0}} \backslash \mathcal{T}$, there exist $\bar{i}$ and $t^{*}$ such that

$$
\begin{gathered}
U_{\bar{i}}\left(t^{*}\right)=W_{\bar{i}}\left(t^{*}-t_{0}\right), \\
U_{i}(t)>W_{i}\left(t-t_{0}\right), \quad t \in\left(t^{*}, t^{*}+\Delta t\right),
\end{gathered}
$$

where $\Delta t>0$ is arbitrarily small. Hence, it follows from (16) and (17) that

$$
D^{+} U_{\bar{i}}\left(t^{*}\right)>D^{+} W_{\bar{i}}\left(t^{*}-t_{0}\right) .
$$

$D^{+} W_{\bar{i}}\left(t^{*}-t_{0}\right)=-\lambda \alpha_{\bar{i}} \bar{\mu}^{N\left(t^{*}, t_{0}\right)}\left[\mathbb{E} V\left(t_{0}\right)\right]^{1 / r} \exp \left(-\lambda\left(t^{*}-t_{0}\right)\right)$.

By the definition of $\lambda$, we have

$$
\begin{aligned}
-\lambda \alpha_{i}> & \frac{1}{r}\left(-\mu_{i} \alpha_{i}+\sum_{j=1}^{n} p_{i j} \alpha_{j}+\sum_{j=1}^{n} q_{i j} \alpha_{j} \exp (\tau \lambda)\right. \\
& \left.+\sum_{j=1}^{n} \int_{0}^{\infty} k_{i j}(s) \exp (\lambda s) \alpha_{j} \mathrm{~d} s\right),
\end{aligned}
$$

which together with (19) yields

$$
\begin{aligned}
& D^{+} W_{\bar{i}}\left(t^{*}-t_{0}\right)>\frac{1}{r}\left(-\mu_{\bar{i}} \alpha_{\bar{i}}+\sum_{j=1}^{n} p_{\bar{i} j} \alpha_{j}+\sum_{j=1}^{n} q_{\bar{i} j} \alpha_{j} \exp (\tau \lambda)+\sum_{j=1}^{n} \int_{0}^{\infty} k_{\bar{i} j}(s) \exp (\lambda s) \alpha_{j} \mathrm{~d} s\right) \\
& \times \bar{\mu}^{N\left(t^{*}, t_{0}\right)}\left[\mathbb{E} V\left(t_{0}\right)\right]^{1 / r} \exp \left(-\lambda\left(t^{*}-t_{0}\right)\right) \\
& =\frac{1}{r}\left(-\mu_{\bar{i}} \alpha_{\bar{i}}\right) \bar{\mu}^{N\left(t^{*}, t_{0}\right)}\left[\mathbb{E} V\left(t_{0}\right)\right]^{1 / r} \exp \left(-\lambda\left(t^{*}-t_{0}\right)\right) \\
& +\frac{1}{r} \sum_{j=1}^{n} p_{i j} \alpha_{j} \bar{\mu}^{N\left(t^{*}, t_{0}\right)}\left[\mathbb{E} V\left(t_{0}\right)\right]^{1 / r} \exp \left(-\lambda\left(t^{*}-t_{0}\right)\right) \\
& +\frac{1}{r} \sum_{j=1}^{n} q_{\overline{i j}} \alpha_{j} \exp (\tau \lambda) \bar{\mu}^{N\left(t^{*}, t_{0}\right)}\left[\mathbb{E} V\left(t_{0}\right)\right]^{1 / r} \exp \left(-\lambda\left(t^{*}-t_{0}\right)\right) \\
& +\frac{1}{r} \sum_{j=1}^{n} \int_{0}^{\infty} k_{i j}(s) \exp (\lambda s) \alpha_{j} \mathrm{~d} s \bar{\mu}^{N\left(t^{*}, t_{0}\right)}\left[\mathbb{E} V\left(t_{0}\right)\right]^{1 / r} \exp \left(-\lambda\left(t^{*}-t_{0}\right)\right) \\
& \geq \frac{1}{r}\left(-\mu_{\bar{i}} \mathbb{E} V_{\bar{i}}\left(t^{*}\right)\right)^{1 / r}+\sum_{j=1}^{n} p_{\bar{i} j}\left(\mathbb{E} V_{\bar{j}}\left(t^{*}\right)\right)^{1 / r} \\
& +\sum_{j=1}^{n} q_{\bar{i} j} \sup _{-\tau \leq \theta \leq 0} \exp (\lambda(\tau+\theta)) \bar{\mu}^{N\left(t^{*}, t^{*}, t_{0}\right)}\left(\mathbb{E} V_{\bar{i}}\left(t^{*}\right)\right)^{1 / r} \\
& \left.+\sum_{j=1}^{n} \int_{0}^{\infty} k_{i j}(s)\left(\mathbb{E} V_{\bar{j}}\left(t^{*}-s\right)\right)^{1 / r} \mathrm{~d} s\right) .
\end{aligned}
$$


Due to $U_{i}(t)=\left[\mathbb{E} V_{i}(t)\right]^{1 / r}$, then $\mathbb{E} V_{i}(t)=\left[U_{i}(t)\right]^{r}$, for $i \in \iota$. By virtue of Dini-derivation, (8), and the Itô formula, we obtain

$$
\begin{aligned}
D^{+} \mathbb{E} V_{i}(t)= & r\left[U_{i}(t)\right]^{r-1} D^{+} U_{i}(t) \\
\leq & -\mu_{i}\left[U_{i}(t)\right]^{r}+\sum_{j=i}^{n} p_{i j}\left[\left(U_{i}(t)\right)\right]^{r / r^{\prime}} U_{j}(t)+\sum_{j=i}^{n} q_{i j} \sup _{-\tau \leq \theta \leq 0}\left[\left(U_{i}(t)\right)\right]^{r / r^{\prime}} U_{j}(t+\theta) \\
& +\sum_{j=i}^{n} \int_{0}^{\infty} k_{i j}(s)\left[\left(U_{i}(t)\right)\right]^{r / r^{\prime}} U_{j}(t-s) \mathrm{d} s .
\end{aligned}
$$

Then, it follows from (22) that

$$
D^{+} U_{i}(t) \leq \frac{1}{r}\left(-\mu_{i} U_{i}(t)+\sum_{j=i}^{n} p_{i j}\left(U_{j}(t)\right)+\sum_{j=i}^{n} q_{i j} \sup _{-\tau \leq \theta \leq 0}\left(U_{j}(t+\theta)\right)+\sum_{j=i}^{n} \int_{0}^{\infty} k_{i j}(s)\left(U_{j}(t-s)\right) \mathrm{d} s\right) .
$$

Combining (21) and (23), we get $D^{+} U_{\bar{i}}\left(t^{*}\right) \leq D^{+} W_{\bar{i}}\left(t^{*}-t_{0}\right)$, which contradicts with (18). Thus, $U_{i}(t) \leq W_{i}\left(t-t_{0}\right)$ holds for all $t \in\left(t_{k}, t_{k+1}\right)$. That is, (13) holds for all $t \in\left(t_{k}, t_{k+1}\right)$.

For Case 2, we have that (13) holds for all $t \in\left[t_{0}, t_{k}\right)$ and does not hold at $t_{k}$. Thus, for some $i \in t$,

$$
\begin{aligned}
& \mathbb{E}\left[V_{i}\left(t_{k}\right)\right] \leq \zeta_{i} \mathbb{E} V_{i}\left(t_{k}^{-}\right)+\left(\sum_{j=1}^{n} o_{i j}\right)\left(\mathbb{E} V_{i}\left(t_{k}^{-}\right)\right)^{1 / r}\left(\mathbb{E} V_{j}\left(t_{k}^{-}\right)\right)^{1 / r} \\
& \leq \zeta_{i} \mathbb{E}\left[V\left(t_{0}\right)\right] \bar{\mu}^{r N\left(t_{k}^{-}, t_{0}\right)} \bar{\alpha}^{r} \exp \left(-r \lambda\left(t_{k}^{-}-t_{0}\right)\right) \\
& +\left(\sum_{j=1}^{n} o_{i j}\right) \times\left(\mathbb{E}\left[V\left(t_{0}\right)\right] \bar{\mu}^{r N\left(t_{k}^{-}, t_{0}\right)} \bar{\alpha}^{r} \exp \left(-r \lambda\left(t_{k}^{-}-t_{0}\right)\right)\right)^{1 / r^{\prime}}\left(\mathbb{E}\left[V\left(t_{0}\right)\right] \bar{\mu}^{r N\left(t_{k}^{-}, t_{0}\right)} \bar{\alpha}^{r} \exp \left(-r \lambda\left(t_{k}^{-}-t_{0}\right)\right)\right)^{1 / r} \\
& \leq\left(\zeta_{i}+\sum_{j=1}^{n} o_{i j}\right) \mathbb{E}\left[V\left(t_{0}\right)\right] \bar{\mu}^{r N\left(t_{k}^{-}, t_{0}\right)} \bar{\alpha}^{r} \exp \left(-r \lambda\left(t_{k}^{-}-t_{0}\right)\right) \\
& \leq \mathbb{E}\left[V\left(t_{0}\right)\right] \bar{\mu}^{r N\left(t_{k}^{-}, t_{0}\right)} \bar{\alpha}^{r} \exp \left(-r \lambda\left(t_{k}^{-}-t_{0}\right)\right),
\end{aligned}
$$

which contradicts with (24). Therefore, (13) holds for $t=t_{k}$.

Therefore, by using the mathematical induction, we see that (13) is satisfied for all $t \in\left[t_{0}, \infty\right)$.

Step 3. Finally, we will prove that system (1) is SGES. In fact, it follows from condition (i) and Jensen's inequality in [29] that

$$
\begin{aligned}
\mathbb{E}[ & \left.V\left(t_{0}\right)\right] \bar{\mu}^{r N\left(t, t_{0}\right)} \bar{\alpha}^{r} \exp \left(-r \lambda\left(t-t_{0}\right)\right) \\
& \leq \mathbb{E}\left[V\left(t_{0}\right)\right] \bar{\mu}^{r N_{0}+r}\left(t-t_{0}\right) / \tau_{c} \bar{\alpha}^{r} \exp \left(-r \lambda\left(t-t_{0}\right)\right) \\
& =\mathbb{E}\left[V\left(t_{0}\right)\right] \bar{\mu}^{r N_{0}} \bar{\alpha}^{r} \exp \left(\left(-r \lambda+r / \tau_{c} \ln \bar{\mu}\right)\left(t-t_{0}\right)\right) \\
& \leq \varsigma \mathbb{E}\left[\|\xi\|_{\tau}\right] \bar{\mu}^{r N_{0}} \bar{\alpha}^{r} n \exp \left(\left(-r \lambda+r / \tau_{c} \ln \bar{\mu}\right)\left(t-t_{0}\right)\right) .
\end{aligned}
$$


According to (iv), we obtain $-r \lambda+\left(r / \tau_{c}\right) \ln \bar{\mu}<0$. This fact together with and (26) gives

$$
\mathbb{E}\left[V_{i}(t)\right] \leq \varsigma \mathbb{E}\left[\|\xi\|_{\tau}\right] \bar{\mu}^{r N_{0}} \bar{\alpha}^{r} n \exp \left(\left(-r \lambda+r / \tau_{c} \times \ln \bar{\mu}\right)\left(t-t_{0}\right)\right) .
$$

By using Markov inequality in [29] and (27), we have that for arbitrary $\varepsilon \in(0,1)$ and $i \in \iota, P\left\{V_{i}(t) \leq \mathcal{\varepsilon}^{-1} \varsigma \mathbb{E}\left[\|\xi\|_{\tau}\right]\right]^{r N_{0}}$ $\left.\bar{\alpha}^{r} n \exp \left(\left(-r \lambda+r / \tau_{c} \times \ln \bar{\mu}\right)\left(t-t_{0}\right)\right)\right\} \geq 1-\varepsilon$, which together with (i) yields

$$
P\left\{|x(t)| \leq \varrho\left(\mathbb{E}\left[\|\xi\|_{\tau}\right], t-t_{0}\right)\right\} \geq 1-\varepsilon,
$$

where $\quad \varrho(a, \quad t)=v^{-1}\left[\mathcal{E}^{-1} \varsigma(a) \mathbb{E}\left[\|\xi\|_{\tau}\right] \bar{\mu}^{r N_{0}} \bar{\alpha}^{r} n \exp ((-r \lambda+\right.$ $\left.\left.\left.r / \tau_{c} \times \ln \bar{\mu}\right) t\right)\right]$. This verifies that system (1) is SGES.
Corollary 1. For system (1), assume that there is locally Lipschitz Lyapunov function $V_{i}: \mathbb{R}_{\geq 0} \times \mathbb{R}^{n} \longrightarrow \mathbb{R}_{\geq 0}$, $i \in l$, $r=1, \quad \zeta E \geq E, \quad v \in \mathscr{K}_{\infty}, \quad \varsigma \in \mathscr{C} \mathscr{K}_{\infty}$, positive matrices $\mu:=\operatorname{diag}\left(\mu_{1}, \mu_{2}, \ldots, \mu_{n}\right), \zeta:=\operatorname{diag}\left(\zeta_{1}, \zeta_{2}, \ldots, \zeta_{n}\right)$, and nonnegative matrices $P:=\left[p_{i j}\right]_{n \times n}, Q:=\left[q_{i j}\right]_{n \times n}, O:=\left[o_{i j}\right]_{n \times n}$, where $K:=\left[\int_{0}^{\infty} k_{i j}(s) d s\right]_{n \times n}$ is continuous function and $k_{i j}(s) \geq 0$ such that the following conditions hold.

$$
\begin{aligned}
& \text { (A): } i \in \iota, t \in \mathbb{R}_{\geq t_{0}}, \nu(|x(t)|) \leq V_{i}(t) \leq \varsigma(|x(t)|) ; \\
& \text { (B): } i \in \iota, t \in \mathbb{R}_{\geq t_{0}} \mid \mathscr{T} .
\end{aligned}
$$

$$
\begin{aligned}
& \mathscr{L} V_{i}\left(t, x_{t}, \int_{0}^{\infty} k(s) x(t-s) \mathrm{d} s\right) \\
& \quad \leq-\mu_{i} V_{i}(t)+\sum_{j=1}^{n} p_{i j} V_{j}(t)+\sup _{-\tau \leq \theta \leq 0} \sum_{j=1}^{n} q_{i j} V_{j}(t+\theta)+\sum_{j=1}^{n} \int_{0}^{\infty} k_{i j}(s) V_{j}(t-s) \mathrm{d} s,
\end{aligned}
$$

where $\mu-P-Q-K$ is a nonsingular M-matrix, $\theta \in[-\tau, 0]$.

(C): $i \in \iota$ and $k \in N_{>0}$,

$$
V_{i}\left(t_{k}\right) \leq \zeta_{i} V_{i}\left(t_{k}^{-}\right)+\left(\sum_{j=1}^{n} o_{i j}\right) V_{j}\left(t_{k}^{-}\right)
$$

(D): $\quad \tau_{c}>\max _{i \in \ell} \ln \left(\zeta_{i}+\sum_{j=1}^{n} o_{i j}\right) / \lambda$, where $\lambda \in(0, \bar{\lambda})$, $V_{i}(t)=V_{i}(t, x(t))$.

$$
\left.\bar{\lambda}:=\sup \left\{\eta>0: \alpha_{i} \eta-\mu_{i} \alpha_{i}+\sum_{j=1}^{n} p_{i j} \alpha_{j}+\sum_{j=1}^{n} q_{i j} \alpha_{j} \exp (\tau \eta)+\sum_{j=1}^{n} \int_{0}^{\infty} k_{i j}(s) \exp (\eta s)\right) \alpha_{j} \mathrm{~d} s<0\right\},
$$

where $i \in l, \alpha \in \Omega_{M}(\mu-P-Q-K)$, with $\min _{1 \leq i \leq n}\left\{\alpha_{i}\right\} \geq 1$, $\bar{\alpha}=\max \left\{\alpha_{i}\right\}$.

Then, system (1) is SGES.

Remark 1. In Theorem 1, a difficulty is that condition $\zeta E>E$ destabilizes system (1). To overcome this difficulty, we give a lower bound by using the relation between the ADT and impulses, and it may guarantee that the number of destabilizing impulses can be reduced. As a consequence, we can prove that system (1) is SGES.
The next theorem will show that the impulses can promote the stability of system (1) even if system (1) without impulses may be unstable.

Theorem 2. For system (1), assume that that there exist locally Lipschitz Lyapunov function $V_{i}: \mathbb{R}_{\geq 0} \times \mathbb{R}^{n} \longrightarrow \mathbb{R}_{\geq 0}$, for all $i \in \iota, r>1, v \in \mathscr{K}_{\infty}, \varsigma \in \mathscr{C} \mathscr{K}_{\infty}$, positive matrices $\mu:=\operatorname{diag}\left(\mu_{1}, \mu_{2}, \ldots, \mu_{n}\right), \zeta:=\operatorname{diag}\left(\zeta_{1}, \zeta_{2}, \ldots, \zeta_{n}\right)$, and nonnegative matrices $P:=\left[p_{i j}\right]_{n \times n}, Q:=\left[q_{i j}\right]_{n \times n}, O:=\left[o_{i j}\right]_{n \times n}$, where $K:=\left[\int_{0}^{\infty} k_{i j}(s) d s\right]_{n \times n}$ be continuous function, $k_{i j}(s) \geq 0$ and $(\zeta+O) E<E$ : 
(A1) $i \in \iota, t \in \mathbb{R}_{\geq t_{0}}, v(|x(t)|) \leq V_{i}(t) \leq \varsigma(|x(t)|) ; \quad$ (A2) $i \in \iota, t \in \mathbb{R}_{\geq t_{0}} \mid \mathscr{T}$.

$$
\begin{aligned}
\mathbb{E} \mathscr{L} V_{i}\left(t, x_{t}, \int_{0}^{\infty} k(s) x(t-s) \mathrm{d} s\right) \leq & \mu_{i} \mathbb{E} V_{i}(t)+\sum_{j=1}^{n} p_{i j}\left(\mathbb{E} V_{i}(t)\right)^{1 / r^{r}}\left(\mathbb{E} V_{j}(t)\right)^{1 / r} \\
& -\sup _{-\tau \leq \theta \leq 0} \sum_{j=1}^{n} q_{i j}\left(\mathbb{E} V_{i}(t)\right)^{1 / r^{r}}\left(\mathbb{E} V_{j}(t+\theta)\right)^{1 / r} \\
& +\sum_{j=1}^{n} \int_{0}^{\infty} k_{i j}(s)\left(\mathbb{E} V_{i}(t)\right)^{1 / r^{r}}\left(\mathbb{E} V_{j}(t-s)\right)^{1 / r} \mathrm{~d} s,
\end{aligned}
$$

where $\mu+P-Q+K$ is a nonsingular M-matrix, $\theta \in[-\tau, 0], r=\left(1-r^{\prime}\right)^{-1}$.

(A3) $i \in \iota$ and $k \in N_{>0}$,

$$
V_{i}\left(t_{k}\right) \leq \zeta_{i} V_{i}\left(t_{k}^{-}\right)+\sum_{j=1}^{n} o_{i j}\left(V_{i}\left(t_{k}^{-}\right)\right)^{1 / r^{r}}\left(V_{j}\left(t_{k}^{-}\right)\right)^{1 / r} .
$$

(A4) $\tau_{c}<\min _{i \in l} \ln \left(\zeta_{i}+\sum_{j=1}^{n} o_{i j}\right) /-\lambda$, where $\lambda>\bar{\lambda}$ and $V_{i}(t)=V_{i}(t, x(t))$.

$$
\bar{\lambda}:=\inf \left\{\omega>0:-r \pi_{i} \omega+\mu_{i} \pi_{i}+\sum_{j=1}^{n} p_{i j} \pi_{j}-\sum_{j=1}^{n} q_{i j} \pi_{j} \exp (\tau \omega)+\sum_{j=1}^{n} \int_{0}^{\infty} k_{i j}(s) \exp (-\omega s) \pi_{j} \mathrm{~d} s<0\right\} .
$$

where $\quad i \in \iota, \quad \pi \in \Omega_{M}(\mu+P-Q+K), \min _{1 \leq i \leq n}\left\{\pi_{i}\right\} \geq 1$, $\bar{\lambda}=\max _{i \in l} \omega_{i}, \bar{\pi}=\max \left\{\pi_{i}\right\}$.

Then, system (1) is SGES.

$$
\mu_{i} \pi_{i}+\sum_{j=1}^{n} p_{i j} \pi_{j}-\sum_{j=1}^{n} q_{i j} \pi_{j}+\sum_{j=1}^{n} \int_{0}^{\infty} k_{i j}(s) \pi_{j} \mathrm{~d} s>0, \quad i \in \iota
$$

Proof. Like the proof of Theorem 1, we divide the proof into three steps.

Step 1. We first prove the existence of $\bar{\lambda}$. Since $\mu+P-$ $Q+K$ is a nonsingular M-matrix, there is

For any given $i$, set $\pi \in \Omega_{M}(\mu+P-Q+K)$, where $\min _{1 \leq i \leq n}\left\{\pi_{i}\right\} \geq 1$, such that

$$
\bar{H}_{i}(\omega)=-r \pi_{i} \omega+\mu_{i} \pi_{i}+\sum_{j=1}^{n} p_{i j} \pi_{j}-\sum_{j=1}^{n} q_{i j} \pi_{j} \exp (\tau \omega)+\sum_{j=1}^{n} \int_{0}^{\infty} k_{i j}(s) \exp (-\omega s) \pi_{j} \mathrm{~d} s .
$$

Obviously, it follows from (36) that $\bar{H}_{i}(0)>0$ and $\bar{H}_{i}(\infty)=-\infty$, where $\omega \longrightarrow \infty$. On the other hand, we have

$$
\begin{aligned}
\bar{H}_{i}^{\prime}(\omega) & =-r \pi_{i}-\tau \sum_{j=1}^{n} q_{i j} \pi_{j} \exp (\tau \omega) \\
& -\sum_{j=1}^{n} \int_{0}^{\infty} s k_{i j}(s) \exp (-\omega s) \pi_{j} \mathrm{~d} s .
\end{aligned}
$$

Then $\bar{H}_{i}^{\prime}(\omega)<0$, and so there is a unique constant $\omega_{i}>0$ such that $\bar{H}_{i}\left(\omega_{i}\right)=0$. Setting $\bar{\lambda}=\min _{1 \leq i \leq n}\left(\omega_{i}\right)$, it is clear that there exists a constant $\lambda>\bar{\lambda}>0$.

Step 2. Next, for $i \in \iota$ and $\lambda>\bar{\lambda}$, then $(\zeta+O) E<E$, we need to prove that

$$
\mathbb{E}\left[V_{i}(t)\right] \leq \mathbb{E}\left[V\left(t_{0}\right)\right] \bar{\mu}^{r N\left(t, t_{0}\right)} \bar{\pi}^{r} \exp \left(r \lambda\left(t-t_{0}\right)\right),
$$


where $V\left(t_{0}\right)=\sum_{i=1}^{n} V_{i}\left(t_{0}\right), \quad \bar{\mu}=\max _{i \in \iota}\left\{\zeta_{i}+\sum_{j=1}^{n} o_{i j}\right\}$, and $\bar{\pi}:=\max _{i \in l}\left(\pi_{i}\right)$. Clearly, (38) holds for $t=t_{0}$.
For convenience, define $W_{i}\left(t-t_{0}\right)=\left[\mathbb{E} V\left(t_{0}\right)\right]^{1 / r}$ $\bar{\mu}^{N\left(t, t_{0}\right)} \pi_{i} \exp \left(\lambda\left(t-t_{0}\right)\right), U_{i}(t)=\left[\mathbb{E} V_{i}(t)\right]^{1 / r}$. It is easy to check that (38) is equivalent to the following:

$$
\left[\mathbb{E} V_{i}(t)\right]^{1 / r} \leq \max _{i \in l}\left\{\left[\mathbb{E} V\left(t_{0}\right)\right]^{1 / r} \bar{\mu}^{N\left(t, t_{0}\right)} \pi_{i} \exp \left(\lambda\left(t-t_{0}\right)\right)\right\}=: \max _{i \in l}\left\{W_{i}\left(t-t_{0}\right)\right\} .
$$

Now suppose that (38) is not true in some interval, then there are two cases:

Case 1. (38) is not true at the non-impulsive time of certain interval;

Case 2. (38) is not true at the impulsive time of certain interval.

For Case 1 , there exists a $k$ such that $U_{i}(t) \leq W_{i}\left(t-t_{0}\right)$ holds for all $t \in\left[t_{0}, t_{k}\right]$ and $i \in l$, and $U_{i}(t) \leq W_{i}\left(t-t_{0}\right)$ is not true for $i \in \iota$ and $t \in\left(t_{k}, t_{k+1}\right)$. Define

$$
t^{*}:=\inf \left\{t \in\left(t_{k}, t_{k+1}\right): U_{i}(t)>W_{i}\left(t-t_{0}\right)\right\} .
$$

Since $U_{i}(t)$ and $W_{i}\left(t-t_{0}\right)$ are continuous for $t \in \mathbb{R}_{\geq t_{0}} \backslash \mathscr{T}$, there exist $\bar{i}$ and $t^{*}$ such that

$$
\begin{aligned}
U_{\bar{i}}\left(t^{*}\right) & =W_{\bar{i}}\left(t^{*}-t_{0}\right), \\
U_{i}(t) & >W_{i}\left(t-t_{0}\right), t \in\left(t^{*}, t^{*}+\Delta t\right) .
\end{aligned}
$$

where $\Delta t>0$ is arbitrarily small. Therefore, it follows from (41) and (42) that

$$
D^{+} U_{\bar{i}}\left(t^{*}\right)>D^{+} W_{\bar{i}}\left(t^{*}-t_{0}\right)
$$

$$
D^{+} W_{\bar{i}}\left(t^{*}-t_{0}\right)=\lambda \pi_{\bar{i}} \bar{\mu}^{N\left(t^{*}, t_{0}\right)}\left[\mathbb{E} V\left(t_{0}\right)\right]^{1 / r} \exp \left(\lambda\left(t^{*}-t_{0}\right)\right) .
$$

By the definition of $\lambda$, we have

$$
\begin{gathered}
\lambda \pi_{i}>\frac{1}{r}\left(\mu_{i} \pi_{i}+\sum_{j=1}^{n} p_{i j} \pi_{j}-\sum_{j=1}^{n} q_{i j} \pi_{j} \exp (\tau \lambda)\right. \\
\left.+\sum_{j=1}^{n} \int_{0}^{\infty} k_{i j}(s) \exp (-\lambda s) \pi_{j} \mathrm{~d} s\right)
\end{gathered}
$$

which together with (44) yields

$$
\begin{aligned}
D^{+} W_{\bar{i}}\left(t^{*}-t_{0}\right)> & \frac{1}{r}\left(\mu_{\bar{i}} \pi_{\bar{i}}+\sum_{j=1}^{n} p_{\bar{i} j} \pi_{j}-\sum_{j=1}^{n} q_{\bar{i} j} \pi_{j} \exp (\tau \lambda)+\sum_{j=1}^{n} \int_{0}^{\infty} k_{\bar{i} j}(s) \exp (-\lambda s) \pi_{j} \mathrm{~d} s\right) \\
& \times \bar{\mu}^{N\left(t^{*}, t_{0}\right)}\left[\mathbb{E} V\left(t_{0}\right)\right]^{1 / r} \exp \left(\lambda\left(t^{*}-t_{0}\right)\right) \\
= & \frac{1}{r}\left(\mu_{\bar{i}} \pi_{\bar{i}}\right) \bar{\mu}^{N\left(t^{*}, t_{0}\right)}\left[\mathbb{E} V\left(t_{0}\right)\right]^{1 / r} \exp \left(\left(\lambda\left(t^{*}-t_{0}\right)\right)\right) \\
& +\frac{1}{r} \sum_{j=1}^{n} p_{\bar{i} j} \pi_{j} \bar{\mu}^{N}\left(t^{*}, t_{0}\right)\left[\mathbb{E} V\left(t_{0}\right)\right]^{1 / r} \exp \left(\lambda\left(t^{*}-t_{0}\right)\right) \\
& -\frac{1}{r} \sum_{j=1}^{n} q_{\bar{i} j} \pi_{j} \exp (\tau \lambda) \bar{\mu}^{N}\left(t^{*}, t_{0}\right)\left[\mathbb{E} V\left(t_{0}\right)\right]^{1 / r} \exp \left(\lambda\left(t^{*}-t_{0}\right)\right) \\
& +\frac{1}{r} \sum_{j=1}^{n} \int_{0}^{\infty} k_{\overline{i j}}(s) \exp (-\lambda s) \pi_{j} \mathrm{~d} s \bar{\mu}^{N}\left(t^{*}, t_{0}\right)\left[\mathbb{E} V\left(t_{0}\right)\right]^{1 / r} \exp \left(\lambda\left(t^{*}-t_{0}\right)\right) \\
\geq & \frac{1}{r}\left(\mu_{\bar{i}}\left(\mathbb{E} V_{\bar{i}}\left(t^{*}\right)\right)^{1 / r}+\sum_{j=1}^{n} p_{\bar{i} j}\left(\mathbb{E} V_{j}\left(t^{*}\right)\right)^{1 / r}\right) \\
& -\frac{1}{r} \sum_{j=1}^{n} q_{\overline{i j}} \sup _{-\tau \leq \theta \leq 0}\left(\mathbb{E} V_{j}\left(t^{*}+\theta\right)\right)^{1 / r} \\
& +\frac{1}{r} \sum_{j=1}^{n} \int_{0}^{\infty} k_{\bar{i} j}(s)\left(\mathbb{E} V_{j}\left(t^{*}-s\right)\right)^{1 / r} \mathrm{~d} s .
\end{aligned}
$$


Due to $U_{i}(t)=\left[\mathbb{E} V_{i}(t)\right]^{1 / r}$, then $\mathbb{E} V_{i}(t)=\left[U_{i}(t)\right]^{r}$, for $i \in \iota$. By virtue of Dini-derivation and the Itô formula, we obtain $D^{+} \mathbb{E} V_{i}(t)=\mathbb{E} \mathscr{L} V_{i}$ and

$$
\begin{aligned}
D^{+} \mathbb{E} V_{i}(t)= & r\left[U_{i}(t)\right]^{r-1} D^{+} U_{i}(t) \leq \mu_{i}\left[U_{i}(t)\right]^{r}+\sum_{j=i}^{n} p_{i j}\left[\left(U_{i}(t)\right)\right]^{r / r^{\prime}} U_{j}(t) \\
& -\sum_{j=i}^{n} q_{i j} \sup _{-\tau \leq \theta \leq 0}\left[\left(U_{i}(t)\right)\right]^{r / r^{\prime}} U_{j}(t+\theta)+\sum_{j=i}^{n} \int_{0}^{\infty} k_{i j}(s)\left[\left(U_{i}(t)\right)\right]^{r / r^{\prime}} U_{j}(t-s) \mathrm{d} s .
\end{aligned}
$$

Therefore, it follows from (47) that

$$
D^{+} U_{i}(t) \leq \frac{1}{r}\left(\mu_{i} U_{i}(t)+\sum_{j=i}^{n} p_{i j}\left(U_{j}(t)\right)-\sum_{j=i}^{n} q_{i j} \sup _{-\tau \leq \theta \leq 0}\left(U_{j}(t+\theta)\right)+\sum_{j=i}^{n} \int_{0}^{\infty} k_{i j}(s)\left(U_{j}(t+s)\right) \mathrm{d} s\right) .
$$

Combining (46) and (48), we get $\left.\left(\mathbb{E} V_{\bar{i}}\left(t^{*}\right)\right)^{1 / r}=D^{+} U_{\bar{i}}\left(t^{*}\right) \leq D^{+} W_{\bar{i}}\left(t^{*}-t_{0}\right)\right)$, which contradicts with (39). In the end, $U_{i}(t) \leq W_{i}\left(t-t_{0}\right)$ holds for all $t \in\left(t_{k}, t_{k+1}\right)$. In other words, (38) holds for all $t \in\left(t_{k}, t_{k+1}\right)$.

For Case 2, (38) holds for all $t \in\left[t_{0}, t_{k}\right.$ ) and dose not hold at $t_{k}$. Then, for some $i \in t$.

$$
\mathbb{E}\left[V_{i}\left(t_{k}\right)\right]>\mathbb{E}\left[V\left(t_{0}\right)\right] \bar{\mu}^{r N\left(t_{k}, t_{0}\right)} \bar{\pi}^{r} \exp \left(r \lambda\left(t_{k}-t_{0}\right)\right) .
$$

By taking expectation and using the Holder inequality, it follows from (A3) that at the impulsive instance $t_{k}$,

$$
\begin{aligned}
& \mathbb{E}\left[V_{i}\left(t_{k}\right)\right] \leq \zeta_{i} \mathbb{E} V_{i}\left(t_{k}^{-}\right)+\left(\sum_{j=1}^{n} o_{i j}\right)\left(\mathbb{E} V_{i}\left(t_{k}^{-}\right)\right)^{1 / r_{1}}\left(\mathbb{E} V_{j}\left(t_{k}^{-}\right)\right)^{1 / r} \\
& \leq \zeta_{i} \mathbb{E}\left[V\left(t_{0}\right)\right] \bar{\mu}^{r N\left(t_{k}^{-}, t_{0}\right)} \bar{\pi}^{r} \exp \left(r \lambda\left(t_{k}^{-}-t_{0}\right)\right)+\left(\sum_{j=1}^{n} o_{i j}\right) \\
& \times\left(\mathbb{E}\left[V\left(t_{0}\right)\right] \bar{\mu}^{r N\left(t_{k}^{-}, t_{0}\right)} \bar{\pi}^{r} \exp \left(r \lambda\left(t_{k}^{-}-t_{0}\right)\right)\right)^{1 / r_{1}}\left(\mathbb{E}\left[V\left(t_{0}\right)\right] \bar{\mu}^{r N\left(t_{k}^{-}, t_{0}\right)} \bar{\pi}^{r} \exp \left(r \lambda\left(t_{k}^{-}-t_{0}\right)\right)\right)^{1 / r} \\
& \leq\left(\zeta_{i}+\sum_{j=1}^{n} o_{i j}\right) \mathbb{E}\left[V\left(t_{0}\right)\right] \bar{\mu}^{r N\left(t_{k}^{-}, t_{0}\right)} \bar{\pi}^{r} \exp \left(r \lambda\left(t_{k}^{-}-t_{0}\right)\right) \\
& \leq \mathbb{E}\left[V\left(t_{0}\right)\right] \bar{\mu}^{r N\left(t_{k}, t_{0}\right)} \bar{\pi}^{r} \exp \left(r \lambda\left(t_{k}-t_{0}\right)\right),
\end{aligned}
$$

which contradicts with (49). This verifies that (38) holds for $t=t_{k}$. Therefore, by using the mathematical induction, we see that (38) is valid for all $t \in\left[t_{0}, \infty\right)$. Step 3. Finally, we will prove that system (1) is SGES. According to $(A 1)$ and Jensen's inequality, we have

$$
\begin{aligned}
\mathbb{E} & {\left[V\left(t_{0}\right)\right] \bar{\mu}^{r N\left(t, t_{0}\right)} \bar{\pi}^{r} \exp \left(r \lambda\left(t-t_{0}\right)\right) } \\
& \leq \mathbb{E}\left[V\left(t_{0}\right)\right] \bar{\mu}^{r N_{0}+r}\left(t-t_{0}\right) / \tau_{c} \bar{\pi}^{r} \exp \left(r \lambda\left(t-t_{0}\right)\right) \\
& =\mathbb{E}\left[V\left(t_{0}\right)\right] \bar{\mu}^{r N_{0}} \bar{\pi}^{r} \exp \left(\left(r \lambda+r / \tau_{c} \ln \bar{\mu}\right)\left(t-t_{0}\right)\right) \\
& \leq \varsigma \mathbb{E}\left[\|\xi\|_{\tau}\right] \bar{\mu}^{r N_{0}} \bar{\pi}^{r} \exp \left(\left(r \lambda+r / \tau_{c} \ln \bar{\mu}\right)\left(t-t_{0}\right)\right) .
\end{aligned}
$$


It follows from condition (A4) that $r \lambda+\left(r / \tau_{c}\right) \ln \bar{\mu}<0$. The rest of the proof is similar to that in Theorem 1, and thus we omit it here. This completes the proof.

Corollary 2. For system (1), assume that there is locally Lipschitz Lyapunov function $V_{i}: \mathbb{R}_{\geq 0} \times \mathbb{R}^{n} \longrightarrow \mathbb{R}_{\geq 0}$, $i \in l$, $r=1,(\zeta+O) E<E, v \in \mathscr{K}_{\infty}, \varsigma \in \mathscr{C}_{K_{\infty}}$, positive matrices $\mu:=\operatorname{diag}\left(\mu_{1}, \mu_{2}, \ldots, \mu_{n}\right), \zeta:=\operatorname{diag}\left(\zeta_{1}, \zeta_{2}, \ldots, \zeta_{n}\right)$, and nonnegative matrices $P:=\left[p_{i j}\right]_{n \times n}, Q:=\left[q_{i j}\right]_{n \times n}, O:=\left[o_{i j}\right]_{n \times n}$.
Where $K:=\left[\int_{0}^{\infty} k_{i j}(s) d s\right]_{n \times n}$ is continuous function and $k_{i j}(s) \geq 0$ such that the following conditions hold:

$$
\begin{aligned}
& \text { (A): } i \in \iota, t \in \mathbb{R}_{\geq t_{0}}, v(|x(t)|) \leq V_{i}(t) \leq \varsigma(|x(t)|) ; \\
& \text { (B): } i \in \iota, t \in \mathbb{R}_{\geq t_{0}} \mid \mathscr{T},
\end{aligned}
$$

$$
\mathscr{L} V_{i}\left(t, x_{t}, \int_{0}^{\infty} k(s) x(t-s) \mathrm{d} s\right) \leq \mu_{i} V_{i}(t)+\sum_{j=1}^{n} p_{i j} V_{j}(t)-\sup _{-\tau \leq \theta \leq 0} \sum_{j=1}^{n} q_{i j} V_{j}(t+\theta)+\sum_{j=1}^{n} \int_{0}^{\infty} k_{i j}(s) V_{j}(t-s) \mathrm{d} s,
$$

where $\mu+P-Q+K$ is a nonsingular M-matrix, $\theta \in[-\tau, 0]$

(C): $i \in \iota$ and $k \in N_{>0}$,

$$
V_{i}\left(t_{k}\right) \leq \zeta_{i} V_{i}\left(t_{k}^{-}\right)+\left(\sum_{j=1}^{n} o_{i j}\right) V_{j}\left(t_{k}^{-}\right)
$$

(D): $\lambda+\left(1 / \tau_{c}\right) \ln \bar{\mu}<0$, where $\bar{\mu}=\max _{i \in t}\left\{\zeta_{i}+\sum_{j=1}^{n} o_{i j}\right\}$ and $\lambda>\bar{\lambda}$ and $V_{i}(t)=V_{i}(t, x(t))$.

$$
\bar{\lambda}:=\inf \left\{\omega>0:-\pi_{i} \omega+\mu_{i} \pi_{i}+\sum_{j=1}^{n} p_{i j} \pi_{j}-\sum_{j=1}^{n} q_{i j} \pi_{j} \exp (\tau \omega)+\sum_{j=1}^{n} \int_{0}^{\infty} k_{i j(s) \exp (-\omega s) \pi_{j} \mathrm{~d} s}<0\right\},
$$

where $i \in l, \pi \in \Omega_{M}(\mu+P-Q+K)$, with $\min _{1 \leq i \leq n}\left\{\pi_{i}\right\} \geq 1$, $\bar{\pi}=\max \left\{\pi_{i}\right\}$.

Then, system (1) is SGES.

Remark 2. In Theorem 2, the condition $(\zeta+O) E<E$ shows that the impulses can do the contribution of the stability of system (1). Although system (1) may not be stable, we can give an upper bound by using the relation between ADT and impulses and prove that SIDSs with DDs and IDDs are SGES.

Remark 3. When $k_{i j}=0, r=2$, Theorems 1 and 2 will be reduced to the case of stochastic differential systems with only DDS, which was studied in [12]. It should be mentioned that [12] only considered time-delay terms coupled with nondelay terms. However, we consider the effect of mixed delay terms including the DDs item and IDDs item coupled with delay-free item, which also appears in the $\mathscr{L} V$-operator differential inequality. Thus, our results not only avoid to use elementary inequality to analyze crossterm problem but also is more representative.

Remark 4. Conditions (ii) and (A2) are the vector version of the Halandy inequality in [9], which is an important tool in the stability analysis of SDSs. Especially, they also play an important role in discussing stochastically perturbed neural networks and stochastically generalized ecological systems.

\section{Two Examples}

In this section, two numerical examples are used to check the validity of our theories.

Consider a two-neuron stochastically perturbed neural network with impulsive control.

$$
\left\{\begin{array}{l}
\mathrm{d} x(t)=\left[A x(t)+B f(x(t))+C f\left(x_{t}\right)+D f\left(\int_{0}^{6} k(s) x(t-s) \mathrm{d} s\right)\right] \mathrm{d} t \\
+\sigma(t, x(t)) \mathrm{d} w(t), t \in \mathbb{R}_{\geq} t_{0} \backslash \mathscr{T}, \\
x\left(t_{k}\right)=h x\left(t_{k}^{-}\right), \quad t_{k} \in \mathscr{T}, k=1,2, \ldots n,
\end{array}\right.
$$


where $x(t) \in \mathbb{R}^{2}, \mathscr{T}$ is a given impulsive time sequence. Define $f(x)=\left(f_{1}\left(x_{1}\right), f_{2}\left(x_{2}\right)\right)=\left(0.05 * \tanh \left(x_{1}\right), 0.05 *\right.$ $\left.\tanh \left(x_{2}\right)\right), x_{t}:=\left(x_{1}(t-\tau), x_{2}(t-\tau)\right)$, then we have

$$
0 \leq\left(f_{i}\left(z_{1}\right)-f_{i}\left(z_{2}\right)\right) /\left(z_{1}-z_{2}\right) \leq 0.05,
$$

for all $z_{1}, z_{2} \in \mathbb{R}$ and $i=1,2$.

\subsection{Example I. Set}

$$
\begin{aligned}
A & =\left[\begin{array}{cc}
-2.4 & 0 \\
0 & -2.8
\end{array}\right], \\
B & =\left[\begin{array}{ll}
1.6 & 3.5 \\
0.3 & 0.4
\end{array}\right], \\
C & =\left[\begin{array}{ll}
0.1 & 0.6 \\
0.4 & 0.1
\end{array}\right], \\
D & =\left[\begin{array}{cc}
0.4 & 0.5 \\
0.3 & 0.1
\end{array}\right], \\
\sigma & =\left[\begin{array}{cc}
0.2 * x_{1}(t) & 0 \\
h & =\left[\begin{array}{cc}
1.5 & 0 \\
0 & 1.5
\end{array}\right],
\end{array}\right.
\end{aligned}
$$

where $k(s)=e^{-s} / 1-e^{-6}$.

Define $\quad V(t, x(t))=\left(V_{1}(t, x(t)), V_{2}(t, x(t))\right) \quad$ with $V_{i}(t, x(t))=\left|x_{i}(t)\right|^{p}, i=1,2(p>1)$. Applying the Itô formula, we get

$$
\begin{aligned}
\mathscr{L} V_{1}\left(t, x_{t}\right) \leq & \left.-2.32 p+0.02\left(p^{2}-p\right)\right)\left|x_{1}(t)\right|^{p} \\
& +0.175 p\left|x_{1}(t)\right|^{p-1}\left|x_{2}(t)\right|+0.05 p\left|x_{1}(t)\right|^{p-1}\left|x_{1}(t-\tau)\right| \\
& +0.03 p\left|x_{1}(t)\right|^{p-1}\left|x_{2}(t-\tau)\right|+0.02 p\left|x_{1}(t)\right|^{p-1} \int_{0}^{6} \frac{e^{-s}}{1-e^{-6}}\left|x_{1}(t-s)\right| \mathrm{d} s \\
& +0.025 p\left|x_{1}(t)\right|^{p-1} \int_{0}^{6} \frac{e^{-s}}{1-e^{-6}}\left|x_{2}(t-s)\right| \mathrm{d} s, \\
\mathscr{L} V_{2}\left(t, x_{t}\right) \leq & \left.-2.78 p+0.02\left(p^{2}-p\right)\right)\left|x_{2}(t)\right|^{p} \\
& +0.015 p\left|x_{2}(t)\right|^{p-1}\left|x_{1}(t)\right|+0.02 p\left|x_{2}(t)\right|^{p-1}\left|x_{1}(t-\tau)\right| \\
& +0.005 p\left|x_{2}(t)\right|^{p-1}\left|x_{2}(t-\tau)\right|+0.015 p\left|x_{2}(t)\right|^{p-1} \int_{0}^{6} \frac{e^{-s}}{1-e^{-6}}\left|x_{1}(t-s)\right| \mathrm{d} s \\
& +\left.0.005 p\left|x_{2}(t)\right|\right|^{p-1} \int_{0}^{6} \frac{e^{-s}}{1-e^{-6}}\left|x_{2}(t-s)\right| \mathrm{d} s V_{i}\left(t_{k}\right) \leq 1.5 V_{i}\left(t_{k}^{-}\right)(i=1,2) .
\end{aligned}
$$


Then, we can easily obtain

$$
\begin{aligned}
\mu & =\left[\begin{array}{cc}
2.32 p-0.02\left(p^{2}-p\right) & 0 \\
0 & 2.78 p-0.02\left(p^{2}-p\right)
\end{array}\right], \\
P & =\left[\begin{array}{cc}
0 & 0.175 p \\
0.015 p & 0
\end{array}\right], \\
Q & =\left[\begin{array}{cc}
00.05 p & 0.03 p \\
0.02 p & 0.05 p
\end{array}\right], \\
K & =\left[\begin{array}{cc}
0.02 p & 0.025 p \\
0.015 p & 0.005 p
\end{array}\right], \\
\zeta & =\left[\begin{array}{cc}
1.5 & 0 \\
0 & 1.5
\end{array}\right] .
\end{aligned}
$$

Thus, we have that $\mu-P-Q-K$ is a nonsingular $M$-matrix, for any $p \in(1,112.4376)$. In particular, when $p=4, \alpha_{1}=1, \alpha_{2}=1.5, \tau=1$, we can calculate $\bar{\lambda}=0.5453$ and $\tau_{c}>2.9743$, which verifies that all the conditions of Theorem 1 are satisfied. Thus, system (1) is SGES. Choosing $\tau_{c}=6$ and a proper $\mathscr{T}$, the initial state $x_{0}=(0.7,-0.6)$, the sample path of solution is given in Figure 1.
4.2. Example II. Set

$$
\begin{aligned}
A & =\left[\begin{array}{cc}
-0.04 & 0 \\
0 & -0.01
\end{array}\right], \\
B & =\left[\begin{array}{ll}
1.6 & 3.5 \\
0.5 & 0.4
\end{array}\right], \\
C & =\left[\begin{array}{cc}
-0.1 & -0.6 \\
-0.4 & -0.1
\end{array}\right], \\
D & =\left[\begin{array}{cc}
1 & 0 \\
0 & 1
\end{array}\right], \\
\sigma & =\left[\begin{array}{cc}
2 * x_{1}(t) & 0 \\
0 & 2 * x_{2}(t)
\end{array}\right], \\
h & =\left[\begin{array}{cc}
0.75 & 0 \\
0 & 0.75
\end{array}\right],
\end{aligned}
$$

where $k(s)=e^{-s} / 1-e^{-6}$.

Define $V(t, x(t))=\left(V_{1}(t, x(t)), V_{2}(t, x(t))\right)$, where $V_{i}(t, x(t))=\left|x_{i}(t)\right|^{p}, i=1,2(p>1)$. Applying the Itô formula, we get

$$
\begin{aligned}
\mathscr{L} V_{1}\left(t, x_{t}\right) \leq & \left(0.04 p+2\left(p^{2}-p\right)\right)\left|x_{1}(t)\right|^{p}+0.175 p\left|x_{1}(t)\right|^{p-1}\left|x_{2}(t)\right| \\
& -0.005 p\left|x_{1}(t)\right|^{p-1}\left|x_{1}(t-\tau)\right|-0.03 p\left|x_{1}(t)\right|^{p-1}\left|x_{2}(t-\tau)\right| \\
& +0.05 p\left|x_{1}(t)\right|^{p-1} \int_{0}^{6} \frac{e^{-s}}{1-e^{-6}}\left|x_{1}(t-s)\right| \mathrm{d} s, \\
\mathscr{L} V_{2}\left(t, x_{t}\right) \leq & \left(0.01 p+2\left(p^{2}-p\right)\right)\left|x_{2}(t)\right|^{p}+0.025 p\left|x_{2}(t)\right|^{p-1}\left|x_{1}(t)\right| \\
& -0.02 p\left|x_{2}(t)\right|^{p-1}\left|x_{1}(t-\tau)\right|-0.005 p\left|x_{2}(t)\right|^{p-1}\left|x_{2}(t-\tau)\right| \\
& +0.05 p\left|x_{2}(t)\right|^{p-1} \int_{0}^{6} \frac{e^{-s}}{1-e^{-6}}\left|x_{2}(t-s)\right| \mathrm{d} s .
\end{aligned}
$$

By taking expectation on both sides of the inequality and using the Holder inequality, we have

$$
\begin{aligned}
\mathbb{E} \mathscr{L} V_{1}\left(t, x_{t}\right) \leq & \left(0.04 p+2\left(p^{2}-p\right)\right) \mathbb{E} V_{1}(t)+0.175 p\left(\mathbb{E} V_{1}(t)\right)^{p-1 / p}\left(\mathbb{E} V_{2}(t)\right)^{1 / p} \\
& -0.005 p\left(\mathbb{E} V_{1}(t)\right)^{p-1 / p}\left(\mathbb{E} V_{1}(t-\tau)\right)^{1 / p}-0.03 p\left(\mathbb{E} V_{1}(t)\right)^{p-1 / p}\left(\mathbb{E} V_{2}(t-\tau)\right)^{1 / p} \\
& +0.05 p\left(\mathbb{E} V_{1}(t)\right)^{p-1 / p} \int_{0}^{6} \frac{e^{-s}}{1-e^{-6}}\left(\mathbb{E} V_{1}(t-s)\right)^{1 / p} \mathrm{~d} s, \\
\mathbb{E} \mathscr{L} V_{2}\left(t, x_{t}\right) \leq & \left(0.01 p+2\left(p^{2}-p\right)\right) \mathbb{E} V_{2}(t)+0.025 p\left(\mathbb{E} V_{2}(t)\right)^{p-1 / p}\left(\mathbb{E} V_{1}(t)\right)^{1 / p} \\
& -0.02 p\left(\mathbb{E} V_{2}(t)\right)^{p-1 / p}\left(\mathbb{E} V_{1}(t-\tau)\right)^{1 / p}-0.005 p\left(\mathbb{E} V_{2}(t)\right)^{p-1 / p}\left(\mathbb{E} V_{2}(t-\tau)\right)^{1 / p} \\
& +0.05 p\left(\mathbb{E} V_{2}(t)\right)^{p-1 / p} \int_{0}^{6} \frac{e^{-s}}{1-e^{-6}}\left(\mathbb{E} V_{2}(t)\right)^{1 / p} \mathrm{~d} s, \\
\mathbb{E} V_{i}\left(t_{k}\right) \leq & 0.75 \mathbb{E} V_{i}\left(t_{k}^{-}\right)(i=1,2) .
\end{aligned}
$$




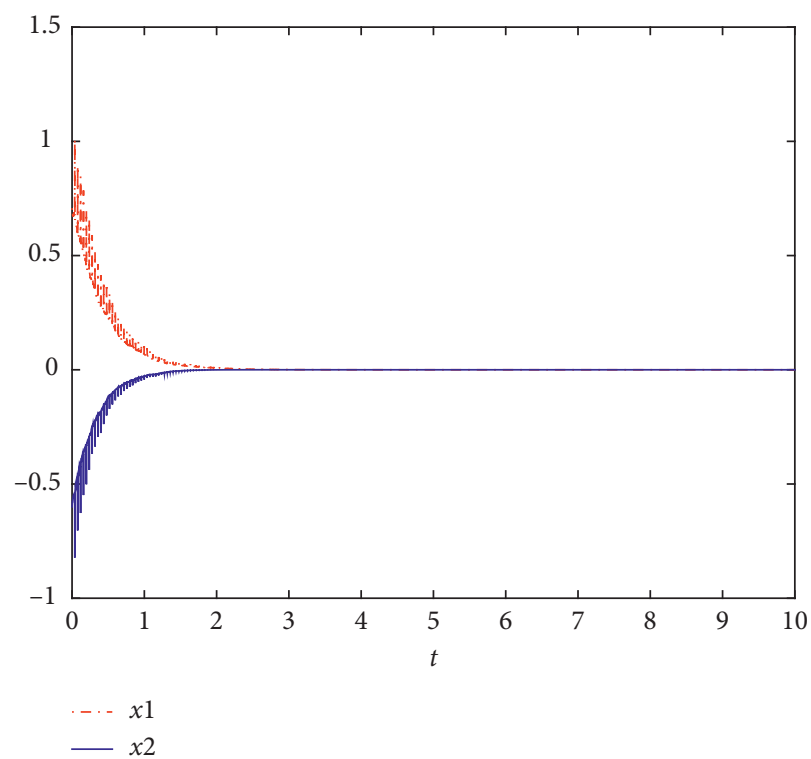

Figure 1: The solution of $\tau=1, \tau_{c}=6$ in example I.

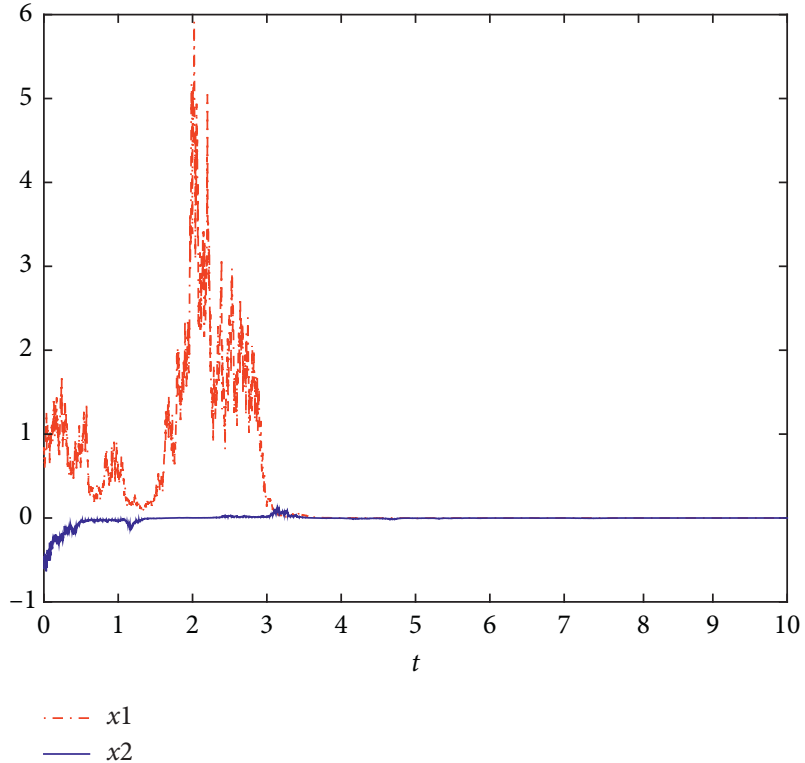

FIgURE 2: The solution of $\tau=1, \tau_{c}=0.16$ in example II.

Then, it follows that

$$
\begin{aligned}
\mu & =\left[\begin{array}{cc}
0.04 p+2\left(p^{2}-p\right) & 0 \\
0 & 0.01 p+2\left(p^{2}-p\right)
\end{array}\right], \\
P & =\left[\begin{array}{cc}
0 & 0.175 p \\
0.025 p & 0
\end{array}\right], \\
Q & =\left[\begin{array}{cc}
0.005 p & 0.03 p \\
0.02 p & 0.005 p
\end{array}\right], \\
K & =\left[\begin{array}{cc}
0.05 p & 0 \\
0 & 0.05 p
\end{array}\right], \\
\zeta & =\left[\begin{array}{cc}
0.75 & 0 \\
0 & 0.75
\end{array}\right] .
\end{aligned}
$$

Then, we see that $\mu+P-Q+K$ is a nonsingular M-matrix for any $p \in(1,+\infty)$. Specially, when $p=2$, $\pi_{1}=1, \pi_{2}=1.5, \tau=1$, we can calculate $\bar{\lambda}=1.9695$ and $\tau_{c}<0.1661$. Thus, all the conditions of Theorem 2 are true, which verifies that (1) is SGES. Choosing $\tau_{c}=0.16$ and a proper $T$, the initial state $x_{0}=(0.7,-0.6)$, the sample path of solution is presented in Figure 2.

\section{Conclusion}

In this paper, we have used the ADT condition and VLF to study SGES of SIDSs with DDs and IDDs under two cases: unstable impulse dynamics situation and stable impulse dynamics situation. By using VLF and ADT conditions, two sufficient stability criteria are established. One is that the lower bound of the mixed system relative to the average dwell-time is SGES when continuous SIDSs with DDs and IDDs is stable and the impulsive effect is unstable. The other is that the impulses can stabilize the system successfully under the upper bound condition of the given average dwell-time when continuous SIDSs with DDs and IDDs could not stochastic stable. Finally, two examples are provided to verify the effectiveness of our results. In future, we will consider apply our method to neural networks [30, 31] and semi-Markov switching systems [32-34].

\section{Data Availability}

No data were used to support this study.

\section{Conflicts of Interest}

The authors declare that they have no conflicts of interest.

\section{Acknowledgments}

This work was jointly supported by the National Natural Science Foundation of China (61773217), the Natural 
Science Foundation of Hunan Province (2020JJ4054), the Hunan Provincial Science and Technology Project Foundation (2019RS1033), and the Scientific Research Fund of Hunan Provincial Education Department (18A013).

\section{References}

[1] S. E. A. Mohammed, Stochastic Functional Differential Equations, Longman Scientific and Technical, London, UK, 1984.

[2] Q. Zhu, "Stability analysis of stochastic delay differential equations with Lévy noise," Systems \& Control Letters, vol. 118, pp. 62-68, 2018.

[3] Q. Zhu and T. Huang, "Stability analysis for a class of stochastic delay nonlinear systems driven by G-Brownian motion," Systems \& Control Letters, vol. 140, Article ID 104699, 2020.

[4] Q. Zhu, "Stabilization of stochastic nonlinear delay systems with exogenous disturbances and the event-triggered feedback control," IEEE Transactions on Automatic Control, vol. 64, no. 9, pp. 3764-3771, 2019.

[5] W. Ma, X. Luo, and Q. Zhu, "Practical exponential stability of stochastic age-dependent capital system with Lévy noise," Systems \& Control Letters, vol. 144, Article ID 104759, 2020.

[6] X. Mao, Stochastic Differential Equations and Their Applications, Elsevier, Amsterdam, Netherlands, 2nd edition, 2007.

[7] M. S. Alwan, X. Liu, and W.-C. Xie, "Existence, continuation, and uniqueness problems of stochastic impulsive systems with time delay," Journal of the Franklin Institute, vol. 347, no. 7, pp. 1317-1333, 2010.

[8] L. Gao, Z. Cao, M. Zhang, and Q. Zhu, "Input-to-state stability for hybrid delayed systems with admissible edge-dependent switching signals," Journal of the Franklin Institute, vol. 357, no. 13, pp. 8823-8850, 2020.

[9] L. Liu, "New criteria on exponential stability for stochastic delay differential systems based on vector Lyapunov function," IEEE Transactions on Systems, Man, and Cybernetics: Systems, vol. 47, no. 11, pp. 2985-2993, 2017.

[10] M. S. Ali, M. Usha, Q. Zhu, and S. Shanmugam, "Synchronization analysis for stochastic T-S fuzzy complex networks with Markovian jumping parameters and mixed time-varying delays via impulsive control," Mathematical Problems in Engineering, vol. 2020, Article ID 9739876, , 2020.

[11] W. Hu and Q. Zhu, "Stability analysis of impulsive stochastic delayed differential systems with unbounded delays," Systems \& Control Letters, vol. 136, Article ID 104606, 2020.

[12] W. Ren and J. Xiong, "Exponential stability of stochastic impulsive switched delayed systems based on vector lyapunov functions," in Proceeding of the 2017 11th Asian Control Conference (ASCC), pp. 1888-1893, Gold Coast, Australia, December 2017.

[13] P. Balasubramaniam and R. Rakkiyappan, "Global asymptotic stability of stochastic recurrent neural networks with multiple discrete delays and unbounded distributed delays," Applied Mathematics and Computation, vol. 204, no. 2, pp. 680-686, 2008.

[14] C. Huang and J. Cao, "Almost sure exponential stability of stochastic cellular neural networks with unbounded distributed delays," Neurocomputing, vol. 72, no. 13-15, pp. 3352-3356, 2009.

[15] G. Chen, D. Li, L. Shi, O. van Gaans, and S. Verduyn Lunel, "Stability results for stochastic delayed recurrent neural networks with discrete and distributed delays," Journal of Differential Equations, vol. 264, no. 6, pp. 3864-3898, 2018.

[16] W. M. Haddad, V. Chellaboina, and S. G. Nersesov, "Impulsive and hybrid dynamical systems: stability, dissipativity and control: stability, dissipativity, and control," in Princeton Series in Applied Mathematics, Princeton University Press, Princeton, NJ, USA, 2014.

[17] W.-H. Chen and W. X. Zheng, "Input-to-state stability and integral input-to-state stability of nonlinear impulsive systems with delays," Automatica, vol. 45, no. 6, pp. 1481-1488, 2009.

[18] J. P. Hespanha, D. Liberzon, and A. R. Teel, "Lyapunov conditions for input-to-state stability of impulsive systems," Automatica, vol. 44, no. 11, pp. 2735-2744, 2008.

[19] S. G. Nersesov and W. M. Haddad, "Control vector Lyapunov functions for large-scale impulsive dynamical systems," Nonlinear Analysis: Hybrid Systems, vol. 1, no. 2, pp. 223-243, 2007.

[20] I. M. Stamova, "Vector Lyapunov functions for practical stability of nonlinear impulsive functional differential equations," Journal of Mathematical Analysis and Applications, vol. 325, no. 1, pp. 612-623, 2007.

[21] W. Ren and J. Xiong, "Stability and stabilization of switched stochastic systems under asynchronous switching," Systems \& Control Letters, vol. 97, pp. 184-192, 2016.

[22] H. Ros, L. Hetel, and D. Efimov, "Vector Lyapunov function based stability for a class of impulsive systems," in Proceedings of the 2015 54th IEEE Conference on Decision and Control, pp. 2247-2251, Osaka, Japan, December 2015.

[23] I. Karafyllis and Z.-P. Jiang, "Global stabilization of nonlinear systems based on vector control Lyapunov functions," IEEE Transactions on Automatic Control, vol. 58, no. 10, pp. 2550-2562, 2013.

[24] J. P. Hespanha and A. S. Morse, "Stability of switched systems with average dwell-time," in Proceedings of the 38th IEEE Conference on Decision and Control, pp. 2655-2660, Phoenix, AZ, USA, December 1999.

[25] R. Bellman, "Vector Lyapunov functions," Journal of the Society for Industrial and Applied Mathematics Series A Control, vol. 1, no. 1, pp. 32-34, 1962.

[26] V. Lakshmikantham, V. M. Matrosov, and S. Sivasundaram, Vector Lyapunov Functions and Stability Analysis of Nonlinear Systems, Springer Science and Business Media, Berlin, Germany, 2013.

[27] P. Zhao, W. Feng, and Y. Kang, "Stochastic input-to-state stability of switched stochastic nonlinear systems," Automatica, vol. 48, no. 10, pp. 2569-2576, 2012.

[28] H. Wang, J. Tan, T. Huang, and S. Duan, "Impulsive delayed integro-differential inequality and its application on IMNNs with discrete and distributed delays," Neurocomputing, vol. 341, pp. 99-106, 2019.

[29] L. Rogers and D. Williams, Diffusions, Markov Processes and Martingales: Volume 2, Itô Calculus, Cambridge University Press, Cambridge, UK, 2000.

[30] Z. Zeng, J. Wang, and X. Liao, "Global exponential stability of a general class of recurrent neural networks with time-varying delays," IEEE Transactions on Circuits and Systems I: Fundamental Theory and Applications, vol. 50, no. 10, pp. 13531358, 2003.

[31] Z. Zeng and J. Wang, "Improved conditions for global exponential stability of recurrent neural networks with timevarying delays," IEEE Transactions on Neural Networks, vol. 17, no. 3, pp. 623-635, 2006.

[32] J. Cheng, J. H. Park, J. Cao, and W. Qi, "A hidden mode observation approach to finite-time SOFC of Markovian 
switching systems with quantization," Nonlinear Dynamics, vol. 100, no. 1, pp. 509-521, 2020.

[33] J. Cheng, D. Zhang, W. Qi, J. Cao, and K. Shi, "Finite-time stabilization of T-S fuzzy semi-Markov switching systems: a coupling memory sampled- data control approach," Journal of The Franklin Institute, vol. 357, no. 16, pp. 11265-11280, 2020.

[34] B. Wang and Q. Zhu, "Stability analysis of semi-Markov switched stochastic systems," Automatica, vol. 94, pp. 72-80, 2018 . 